\title{
A Comparative Performance Analysis of Channel Equalizer in OFDM System Under Jamming Attacks
}

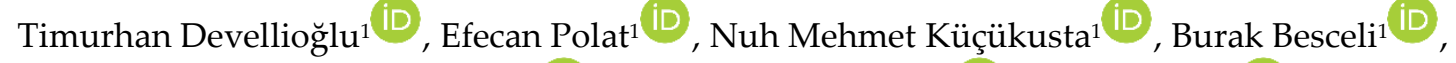 \\ Enver Faruk Tanrıkulu1 ${ }^{1}$, Mehmet Murat Güçlü ${ }^{1}$ and Ali Özen ${ }^{1+}$ iD \\ ${ }^{1}$ Department of Electrical and Electronics Engineering, Nuh Naci Yazgan University - HARGEM \\ Kayseri, Turkey \\ (timurhand38@gmail.com, polatefe64@gmail.com, mkkucukusta@gmail.com, \\ burakbesceli@gmail.com, enverfaruktanrikulu@gmail.com, mguclu.tr@gmail.com, aozen@nny.edu.tr) \\ *1 (aozen@nny.edu.tr)
}

Received: Sep.3, 2021

Accepted: Sep.16,2021

Published: Oct.20, 2021

\begin{abstract}
In this article, the accomplishment of OFDM systems under different jamming attacks is analyzed. Partial band jamming signal suppressing OFDM data packets partially and full band jamming signal suppressing all OFDM data packets are examined in this study. Computer simulation works are carried out to analyze the achievement of the OFDM system over AWGN and frequency selective Rayleigh fading channels under both jamming attacks. In addition, comparative performance analysis of different equalizer structures in frequency selective Rayleigh fading channel environment is performed. From the obtained results, it is seen that frequency domain equalizers combat jamming attack signals in the best way and provide the best performances.
\end{abstract}

Keywords: OFDM, jamming attack signals, partial band jamming, full band jamming, OFDM-FDE, OFDMTDE.

\section{Introduction}

Wireless communication, which is an indispensable part of our lives, has become a widely used technology. Mobile communication systems, which are one of the many wireless communication application areas today, provide many services to the user such as voice, SMS, image transfer, video transfer, internet access (Baltac1, 2018).

The widespread use of mobile phones makes communication and life easier, while threatening information security (national or commercial) corporate-public interests; It threatens exam security in schools, travel safety in intercity buses, peace and security of trials in courtrooms, and security in power plants. The problems caused by mobile phones in the cardiology clinics of hospitals are at a very serious level. Remotely controlled bombs can be activated by mobile phones. Telephones are used as a means of wiretapping, public information is polluted in the cheapest and simplest way, and private companies' information is accessed. Both private and institutional sensitivity to this dangerous pollution has increased in the direction of preventing the use of mobile phones in certain areas (Baltacl, 2018).

The purpose of the scrambling process is to prevent the target system from performing its functions completely or temporarily. Confusion of cellular communication systems can be defined as the 
operations performed to prevent the users from getting service from the mobile terminal (Kaplan et al., 2020; Alakoca et al., 2016).

Scrambling methods target certain channels in the mobile terminal-base station interface. Thus, the data on the receiver side is tried to be made unrecognizable ( $\mathrm{Li}$ et al., 2017).

Orthogonal Frequency Division Multiplexing (OFDM) is an encouraging scheme that allows high data rates to be transmitted. The basic idea of OFDM is to utilize multiple parallel narrowband subcarriers to carry information instead of a single broadband carrier. OFDM can efficiently give wide band wireless telecommunication in hostile multipath surroundings, thanks to its ability to adapt to harsh channel conditions without complex equalization. In addition, OFDM is resistant to Inter-Symbol Interference (ISI) and damping caused by multi-path spreading.

As OFDM is a very significant nominee for the core technology of next generation wireless telecommunication schemes, it is vital to investigate its success under deliberate interference over the weakening channel. There are some studies in this subject. In (Kaplan et al., 2020), the accomplishment of OFDM telecommunication in the presence of partial band mixing is exhibited. The anti-jamming attribute of clustered OFDM has been investigated in (Alakoca et al., 2016), and (Li et al., 2017) gives a detailed work on the partial band scrambling effect on OFDM schemes. Notwithstanding all the revealed studies, there has not been a comprehensive research on the influences of distinctive mixing schemes for OFDM technologies. By evaluating the Bit Error Rate (BER) performance of distinctive scrambling methods, the most efficient scrambling method can be determined under several channel conditions. This is important for OFDM systems, both for mixing and anti-mixing implementations (Liu et al., 2010; Soliman et al., 2018).

In this article, the effects of different jamming strategies such as partial band jamming (PBJ) and full band jamming (FBJ) on the BER achievement of the equalizers used in the OFDM scheme in the additive white Gaussian noise (AWGN) channel and frequency selective Rayleigh fading channel environments are evaluated.

Jamming signal models utilized in studies are summarized in Section 2. Chapter 3 introduces the OFDM system under jamming attack signals. In the fourth Section, computer simulation environment and studies are introduced. In the last part, the acquired outcomes by considering the computer simulation results are interpreted.

\section{Jamming Signal Models}

Two types of jamming attack signals are used in this study. The first is a partial band jamming signal and the other is a full band jamming signal.

\subsection{Partial Band Jamming Signal}

Partial band jamming signal is shown in Figure 1.

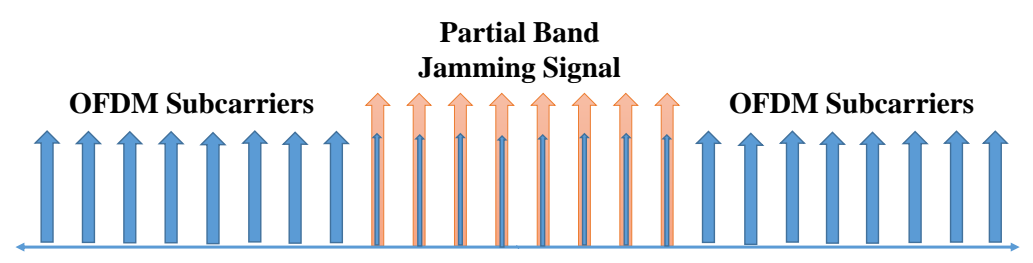

Figure 1. Partial band jamming signal (Devellioğlu, 2021).

As seen in Figure 1, the distorting power of the partial band jamming signal is evenly distributed over a part of the whole frequency band of OFDM sub-carriers, and the frequency spectrum of the received OFDM signal has two jamming and non-jamming parts. In this study, as can be seen from the figure, a jamming signal is applied to the $4 \mathrm{MHz}$ band of the OFDM signal with a bandwidth of $20 \mathrm{MHz}$. 


\subsection{Full Band Jamming Signal}

The full band jamming signal is shown in Figure 2.

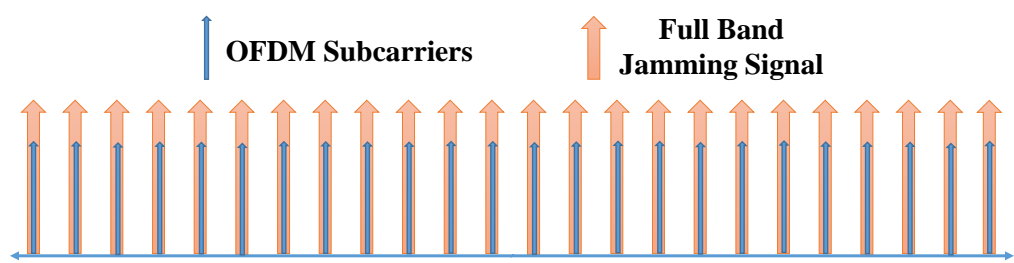

Figure 2. Full band jamming signal (Devellioğlu, 2021).

As seen in Figure 2, the disruptive power of the full band jamming signal, which is the simplest jamming attack kind, is equally dispersed over the entire frequency domain of OFDM sub-carriers. In this study, as can be seen from the figure, a jamming signal is applied to the $20 \mathrm{MHz}$ band of the OFDM signal with a bandwidth of $20 \mathrm{MHz}$.

\section{OFDM System with Jamming Attack Signal}

Figure 3 illustrates the transmitter receiver unit scheme of an OFDM system under the influence of the jamming attack signal (Devellioğlu, 2021).
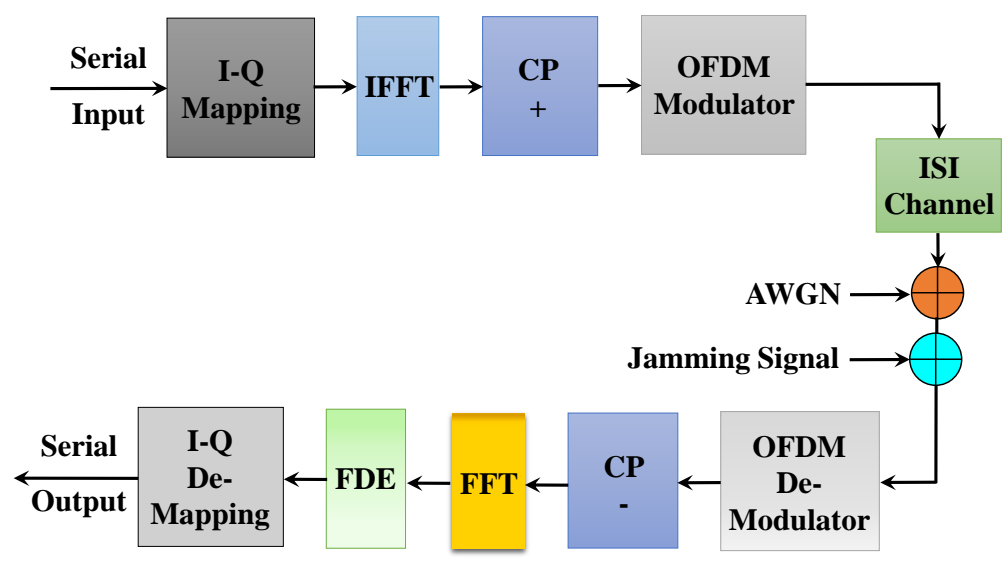

Figure 3. Block scheme of OFDM transceiver under jamming attack signal (Devellioğlu, 2021).

On the transmitter part of the OFDM scheme given in Figure 3, randomly produced serial input information bits are subjected to the inverse fast Fourier transform (IFFT) process after being modulated in the I-Q Mapping Unit. An OFDM symbol package is then created by adding the cyclic prefix (CP) in the $\mathrm{CP}+$ block. The generated OFDM data packets are transmitted via the frequency selective Rayleigh fading channel. Later, OFDM data packets deformed by additive white Gaussian noise (AWGN) and jamming attack signals reach to the receiver.

The generation of jamming attack signals in this study is the same as the generation of OFDM signals as described above.

On the receiving part, the transmitted information bits are estimated by doing the opposite of the procedures on the transmitter part. Then the desired performance criteria are calculated. In this study, time domain and frequency domain channel equalizers are used on the receiver part to restore the distorted information bits. 


\section{Computer Simulation Studies}

The simulation studies of computer are composed of two stage. In the first part, AWGN channel simulations under jamming attack signals and in the second stage, frequency selective Rayleigh fading channel simulations are made under jamming attack signals. 64-point FFT process and 16-length $\mathrm{CP}$ are used in all of the studies.

\subsection{AWGN Channel Simulation Results Under Jamming Attack Signals}

In this section, AWGN channel simulations of the OFDM system are made in the existence of partial band jamming attack and full band jamming attack signals. Simulations are performed via 1000 Monte Carlo channel loops for 100 OFDM data frames with BPSK and 4-QAM modulated signals.

In Figure 4 , as the signal jamming ratio is $\mathrm{SJR}=20 \mathrm{~dB}$, the AWGN channel performances of the BPSK and 4-QAM module OFDM system are given under both jamming attack signals.

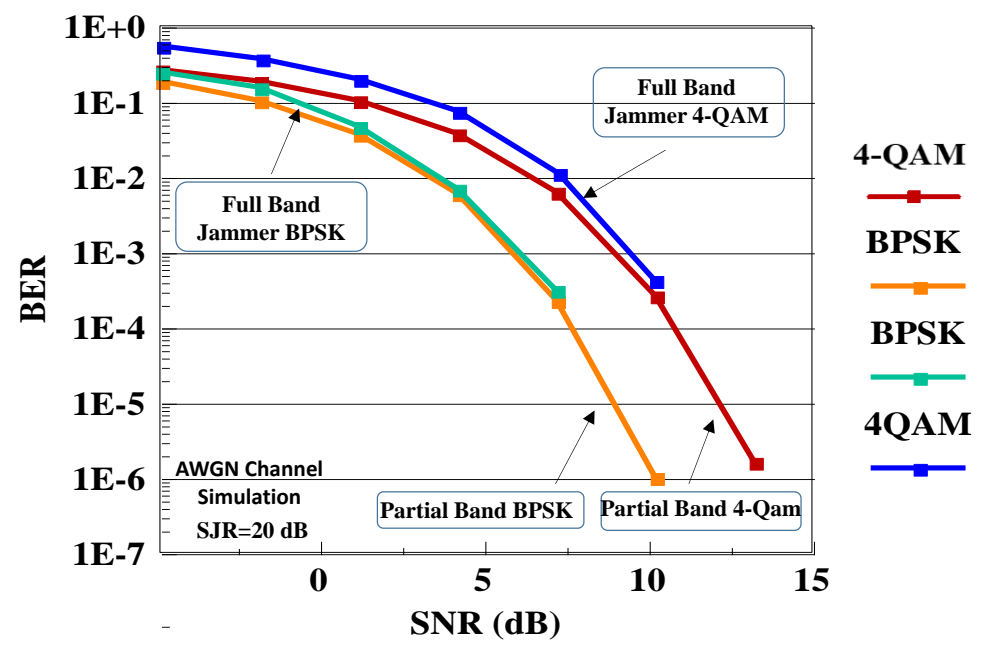

Figure 4. BER performance comparison of OFDM system under different jamming attack signal for $\mathrm{SJR}=20 \mathrm{~dB}$.

As Figure 4 is evaluated, it is perceived that the acquired accomplishments in the presence of both Jamming attack signals in the AWGN channel are approximately the same. A difference of approximately $2 \mathrm{~dB}$ between BPSK and 4-QAM modulation is observed in the Figure.

In Figure 5, while the signal jamming ratio is $\mathrm{SJR}=10 \mathrm{~dB}$ and $30 \mathrm{~dB}$, the AWGN channel performances of the BPSK and 4-QAM modulated OFDM system are given under the full band jamming attack signal. 


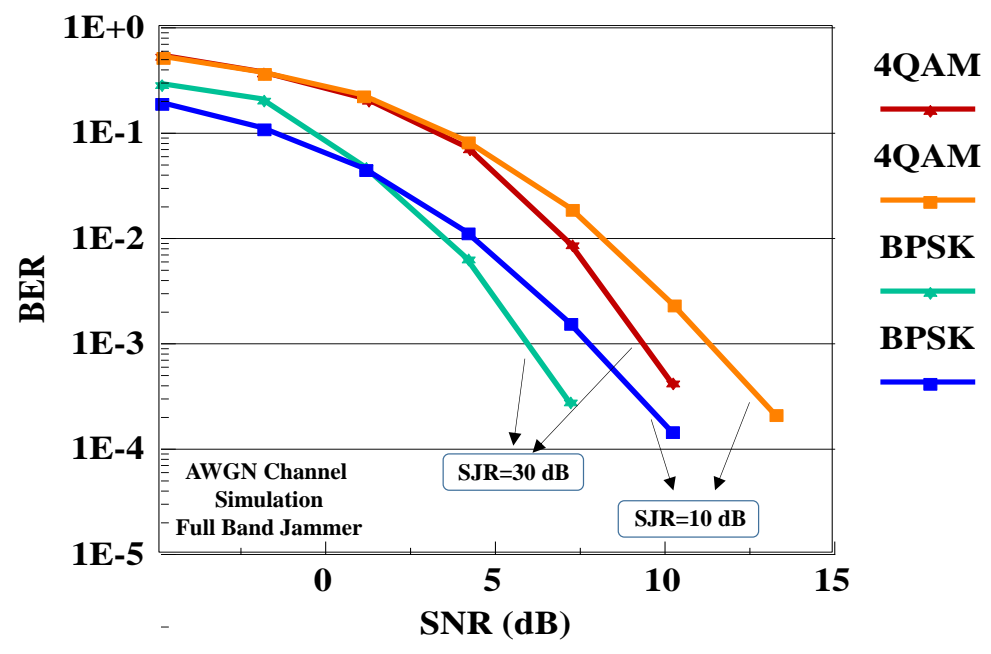

Figure 5. BER accomplishment comparison of OFDM scheme under full band jamming attack signal for $S J R=10 \mathrm{~dB}$ and $30 \mathrm{~dB}$.

As Figure 5 is evaluated, it is noticed that the acquired achievements in the presence of full band Jamming attack signal in the AWGN channel improve as the SJR value increases. A difference of approximately $3 \mathrm{~dB}$ between BPSK and 4-QAM modulation is observed in the Figure.

\subsection{Frequency Selective Rayleigh Fading Channel Simulation Results Under Jamming Attack Signals}

In this section, frequency selective Rayleigh fading channel simulations of OFDM scheme are performed in the existence of partial band jamming attack and full band jamming attack signals. Simulations are performed via 1000 Monte Carlo channel loops for 100 OFDM data frames with BPSK and 4-QAM modulated signals. Time domain and frequency domain equalizers are used to equalize the 5-taps frequency selective Rayleigh fading channel. As the time domain equalizer (TDE), 13 taps channel matched filter decision feedback equalizer (CMF-DFE), which consists of 9-taps feed forward filter and 4-taps feedback filter, is utilized. As frequency domain equalizer, classical frequency domain equalizer (FDE) and FDE, where channel coefficients are estimated in the time domain and equalized in the frequency domain, are used. In the simulation studies in this section, it is supposed that the channel coefficients are known on the receiver part.

In Figure 6, while the signal jamming ratio is $S J R=10 \mathrm{~dB}$ and $30 \mathrm{~dB}$, the frequency selective Rayleigh fading channel performances of the BPSK modulated OFDM system under the partial band jamming attack signal are given. 


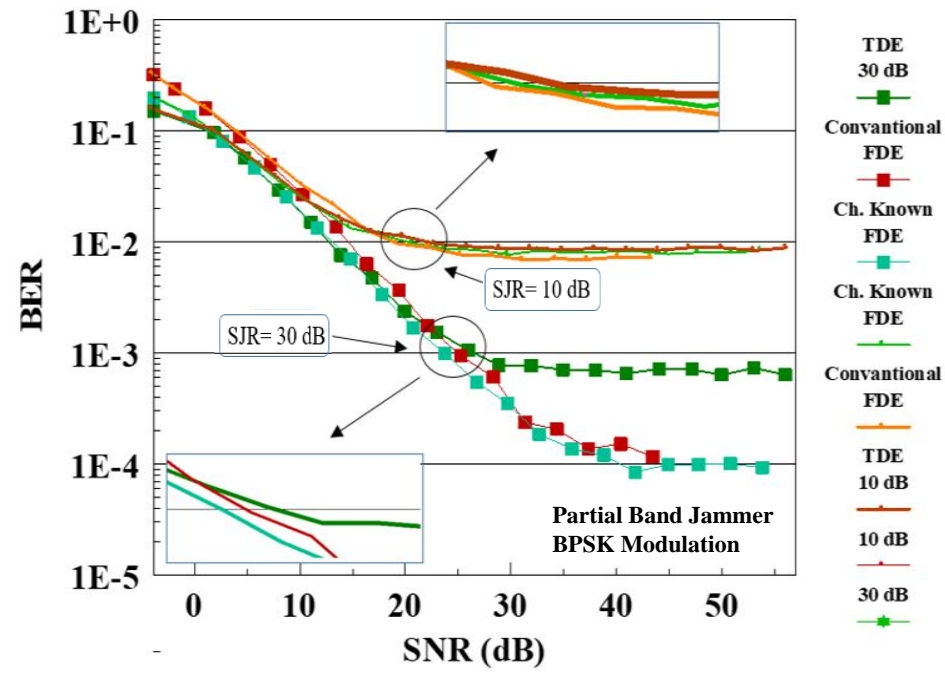

Figure 6. BER performance comparison of OFDM system under partial band jamming attack signal for $\mathrm{SJR}=10 \mathrm{~dB}$ and $30 \mathrm{~dB}$ in BPSK modulated signals.

As Figure 6 is inspected, it is perceived that the acquired accomplishment with TDE for SJR $=30 \mathrm{~dB}$ converges to 7E-4 BER after $30 \mathrm{~dB}$ SNR. On the other hand, by passing the TDE performance with both FDEs, performances converging approximately to $1 \mathrm{E}-4 \mathrm{BER}$ value after $35 \mathrm{~dB}$ SNR value are obtained. In addition, it is seen from the Figure that as the SJR value increases, the performances improve.

In Figure 7, while the signal jamming ratio is $S J R=10 \mathrm{~dB}$ and $30 \mathrm{~dB}$, the frequency selective Rayleigh fading channel performances of the 4-QAM modulated OFDM system under the partial band jamming attack signal are given.

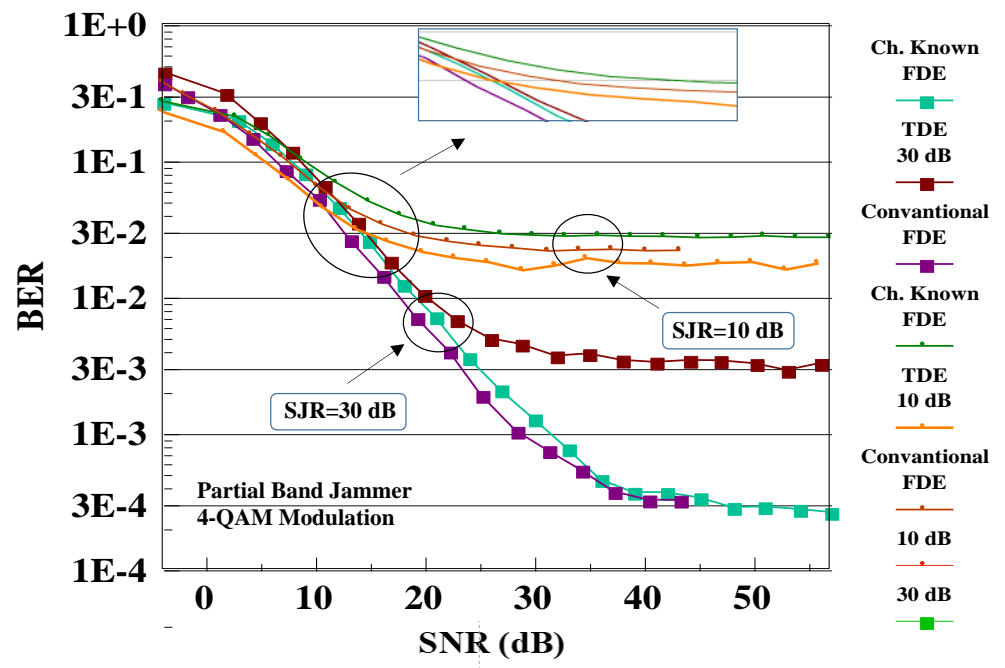

Figure 7. BER performance comparison of OFDM system under partial band jamming attack signal for $\mathrm{SJR}=10 \mathrm{~dB}$ and $30 \mathrm{~dB}$ in 4-QAM modulated signals.

As Figure 7 is investigated, it is noticed that the acquired performance with TDE for SJR $=30 \mathrm{~dB}$ converges to 3E-3 BER value after $30 \mathrm{~dB}$ SNR value. On the other hand, by passing the TDE performance with both FDEs, performances converging approximately to 3E-4 BER value after $40 \mathrm{~dB}$ SNR value are obtained. In addition, it is seen from the Figure that the performances worsen compared to the previous results as the modulation depth increases.

In Figure 8, while the signal jamming ratio is $\mathrm{SJR}=10 \mathrm{~dB}$ and $30 \mathrm{~dB}$, the frequency selective Rayleigh fading channel performances of the BPSK modulated OFDM system under the full band jamming attack signal are given. 


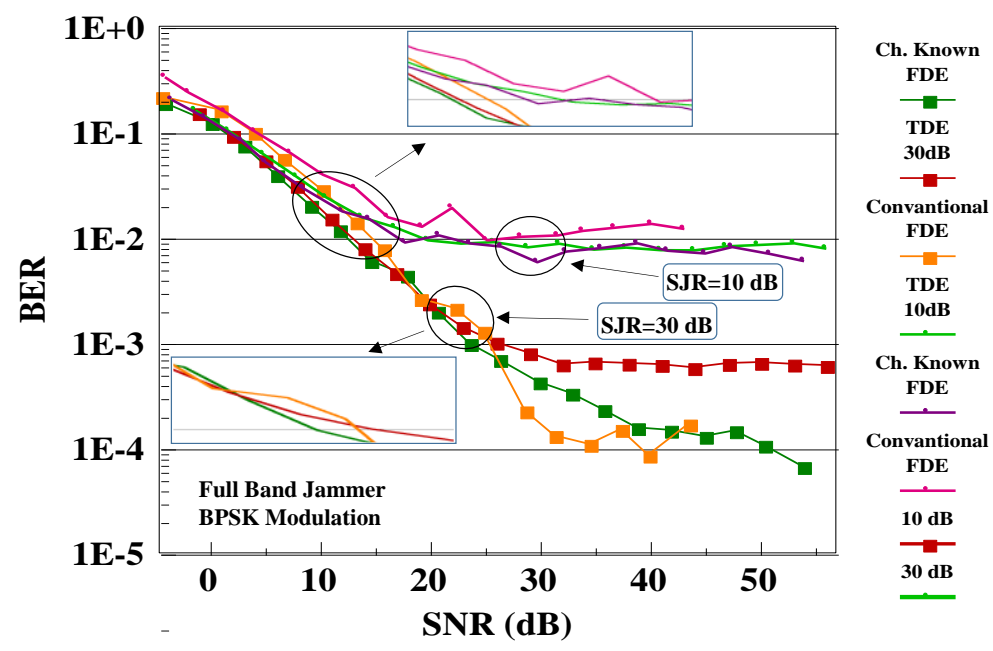

Figure 8. BER performance comparison of OFDM system under full band jamming attack signal for $\mathrm{SJR}=10 \mathrm{~dB}$ and $30 \mathrm{~dB}$ in BPSK modulated signals.

As Figure 8 is evaluated, it is perceived that the acquired achievement with TDE for SJR $=30 \mathrm{~dB}$ converges to $6 \mathrm{E}-4 \mathrm{BER}$ value after $30 \mathrm{~dB}$ SNR value. On the other hand, by passing the TDE performance with both FDEs, performances converging approximately to $1 \mathrm{E}-4 \mathrm{BER}$ value after $30 \mathrm{~dB}$ SNR value are obtained. In addition, it is seen from the Figure that as the SJR value increases, the performances improve.

In Figure 9, while the signal jamming ratio is $\mathrm{SJR}=10 \mathrm{~dB}$ and $30 \mathrm{~dB}$, the frequency selective Rayleigh damped channel performances of the 4-QAM modulated OFDM system under the full band jamming attack signal are given.

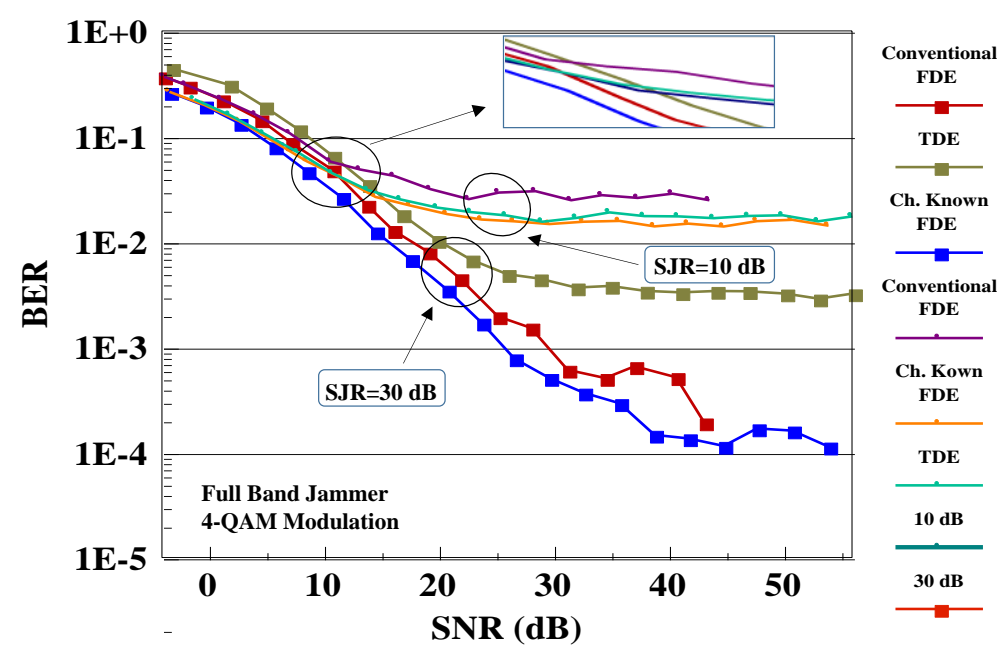

Figure 9. BER performance comparison of OFDM system under full band jamming attack signal for $\mathrm{SJR}=10 \mathrm{~dB}$ and $30 \mathrm{~dB}$ in $4-\mathrm{QAM}$ modulated signals.

As Figure 9 is evaluated, it is noticed that the acquired accomplishment with TDE for SJR $=30 \mathrm{~dB}$ converges to $4 \mathrm{E}-3 \mathrm{BER}$ after $30 \mathrm{~dB}$ SNR level. On the other hand, it is perceived that TDE performance is exceeded with both FDEs. However, it is observed that the FDE, where the channel coefficients are estimated in the time domain and equalized in the frequency domain, exceeds the classical FDE. In addition, it is understood from the Figure that the performances worsen compared to the previous results as the modulation depth increases. 


\section{Conclusion}

In this study, the effects of partial band and full band jamming attack signals, which are among the jamming attack signal types, on the performance of OFDM schemes were evaluated. In order to observe the effects of the related jamming attack signals on OFDM schemes, computer simulations were executed over AWGN and frequency selective Rayleigh fading channels. Equalizer structures used in OFDM systems in frequency selective Rayleigh fading channel environments are compared. The obtained results showed that frequency domain equalizers are better than time domain equalizers.

\section{References}

Alakoca H., Tugrel H. B., Kurt G. K. and Ayyıldız C. (2016), CP and Pilot Jamming Attacks on SCFDMA: Performance Tests with Software Defined Radios, 2016 10th International Conference on Signal Processing and Communication Systems (ICSPCS), 1-6.

Baltacı A. (2018), Effects of hardware and design parameters of adaptive digital predistortion on system performance, Master of Science Thesis, Hacettepe University.

Devellioğlu T. (2021), Investigation of the effect of Jamming Attack signals on Multi Carrier Communication Systems, Bachelor Degree Thesis, Nuh Naci Yazgan University - HARGEM, Kayseri, TURKEY, June 2021.

Kaplan A., Altunbaş İ., Kurt G. K., Kesal M. and Küçükyavuz D. (2020), OFDM-IM Performance Evaluation Under Jamming Attack, 2020 30th International Telecommunication Networks and Applications Conference (ITNAC), 1-6.

Li L., Tian S., Huang J. and Zhang P. (2017), Efficient and Unrecognizable OFDM Jamming By Failing the Cyclic Prefix Functionality, 2017 9th International Conference on Wireless Communications and Signal Processing (WCSP), 1-6.

Liu Y., Ning P., Dai H. and Liu A. (2010), Randomized Differential DSSS: Jamming-Resistant Wireless Broadcast Communication, 2010 Proceedings IEEE INFOCOM, 1-9.

Soliman M. A., Saad W. M., Moussa K. H., Shaaban S. and Rizk M. R. M. (2018), Analysis of Jamming Attacks on a Hopped OFDM Communication System, 2018 18th IEEE International Conference on Communication Technology, 407-411. 